\title{
Efectividad de la vacuna antivaricela como profilaxis post exposición en niños chilenos
}

\author{
Constanza Pinochet, Jaime Cerda, Tamara Hirsch, \\ Jocelyn Mieres, Carolina Inostroza y Katia Abarca
}

\section{Effectiveness of varicella vaccine as post exposure prophylaxis in Chilean children}

Introduction: Efficacy and effectiveness of varicella vaccine (VV) as post exposure prophylaxis (PEP) strategy have shown discordant results for disease risk reduction, and more consistent results in their ability to attenuate the disease. Aim: To assess the effectiveness of VV as PEP among household contacts. Material and Methods: We followed up 33 children after household exposure to a varicella case, of which 15 received VV as PEP and 18 did not received VV. The presence and severity of the disease were clinically determined. Results: Secondary attack rate was 53\% among vaccinated and 89\% among non-vaccinated children. Overall effectiveness of VV as PEP was 40\% (CI95\% 1\%-64\%). The effectiveness for preventing moderate or severe disease was 63\% (CI95\% 8\%-85\%) in the entire group and $77 \%$ (CI95\% 14\%-94\%) among children vaccinated during the first 3 days post exposure. Vaccine tolerance was acceptable, with a low number of adverse reactions, all of them mild. Conclusion: The results suggest that VV as PEP is effective among household contacts, especially for reducing the severity of the disease.

Key words: Varicella, varicella vaccine, post exposure prophylaxis.

Palabras clave: Varicela, vacuna antivaricela, profilaxis post exposición.

\section{Introducción}

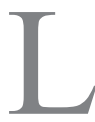

a varicela corresponde al síndrome clínico consecutivo a la primoinfección por virus varicela zóster. De curso generalmente benigno, se describen complicaciones tales como infecciones bacterianas de piel y tejidos blandos, meningoencefalitis y cerebelitis, neumonía, síndrome purpúrico, hepatitis y artritis reactiva ${ }^{1}$. Las complicaciones pueden resultar en secuelas estéticas, neurológicas y ortopédicas, y en los casos más graves pueden producir la muerte ${ }^{1,2}$. Su modo de transmisión es de persona a persona tanto por contacto directo con mucosas infectadas como por partículas aéreas de secreciones respiratorias, y también por vía transplacentaria. Cuando un contacto susceptible (i.e. sin historia de enfermedad ni vacunación) se expone a un caso de varicela, la tasa de ataque secundario fluctúa entre 78 y $92 \%$. Dentro de un hogar los casos secundarios suelen ser más graves, hecho que podría estar relacionado con una mayor carga de virus infectante al momento del contagio $^{1,3}$.

Desde mediados de 1980 se dispone de la vacuna antivaricela (VAV). Su eficacia contra toda enfermedad es de $70-80 \%$ y contra enfermedad moderada a intensa es de $95-100 \%$, siendo su administración recomendada por la Academia Americana de Pediatría a partir del año de edad ${ }^{6}$. A pesar de que la vacuna es bien tolerada, se han reportado efectos adversos tales como fiebre (14\%), alteraciones en el sitio de inyección (15-20\%) y rash vesiculoso $(4 \%)^{5}$. Como profilaxis post exposición (PPE) para varicela existen distintas alternativas: inmunoprofilaxis pasiva con inmunoglobulina hiperinmune anti virus varicela zóster (de uso restringido a pacientes de alto riesgo), aciclovir oral (recomendado en la segunda semana post contacto $)^{4,7-9}$, y vacunación post exposición, siendo esta última recomendada por el Advisory Committee on Immunization Practices (ACIP) por sobre el uso de aciclovir $^{10}$. En la literatura médica, el reporte de efectividad de la VAV como PPE varía entre 9 y $100 \%{ }^{11-21}$. Al respecto, una revisión sistemática realizada por la Colaboración Cochrane (2008) identificó y consideró metodológicamente analizables solamente tres ensayos clínicos aleatorios de eficacia de la VAV como PPE en niños sanos con contacto intradomiciliario, concluyendo que existe evidencia limitada para indicar su uso debido al número pequeño de participantes en los estudios y los variados resultados obtenidos $^{3,17-19}$. Los tres estudios incluidos contaban con un grupo de niños vacunados y un grupo control (novacunados). Dos de ellos reportaron mayor incidencia de varicela en no-vacunados $v s$ vacunados ${ }^{17,18}$, mientras que un tercer estudio no reportó diferencias entre ambos grupos ${ }^{19}$. Los tres estudios fueron consistentes en señalar que aquellos vacunados que enferman casi en su totalidad presentan varicela leve $\mathrm{e}^{17-19}$.

En Chile, la VAV no se encuentra incluida en el Programa Nacional de Inmunizaciones, ni tampoco existe una recomendación emanada del Ministerio de Salud respecto a su uso como PPE. A la fecha no existen
Pontificia Universidad Católica de Chile.

Facultad de Medicina, División de Pediatría (CP, TH, JM, CI, KA). Facultad de Medicina,

Departamento de Salud Pública (JC)

Declaración de conflictos de interés: K. Abarca ha recibido financiamiento de GSK y Sanofi Pasteur para estudios clínicos de vacunas, asistencia a congresos, cursos y dictar conferencias; ha recibido honorarios por participar en comité de expertos en vacunas por GSK. T. Hirsch ha recibido financiamiento para asistir a congresos y dictar cursos de GSK. El resto de los autores declara no presentar conflictos de interés.

Fuente de financiamiento: Proyecto financiado con fondos de la División de Pediatría de la Pontificia Universidad Católica de Chile, obtenidos por concurso.

Recibido: 8 de mayo de 2012 Aceptado: 18 de octubre de 2012

Correspondencia a: Katia Abarca V. katia@med.puc.cl 
reportes nacionales acerca de su uso como PPE. Por consiguiente, el objetivo del presente estudio fue determinar la efectividad de la VAV en niños expuestos a un caso índice domiciliario, aportando nueva evidencia que apoye la toma de decisiones. Como hipótesis de investigación, planteamos que la administración de VAV como PPE reduce la incidencia de enfermedad en contactos domiciliarios de casos de varicela.

\section{Materiales y Métodos}

\section{Tipo de estudio y participantes}

Estudio de cohorte concurrente. Se consideró como exposición el uso de VAV como PPE en sujetos susceptibles, y como evento de interés la ocurrencia de varicela, verificada clínicamente. Durante un período de 15 meses fueron reclutados niños de 1 a 12 años de edad susceptibles a la infección, correspondientes a contactos domiciliarios de pacientes que consultaron por varicela en centros ambulatorios de la Red de Salud UC. Se definió como contacto domiciliario a niños que habitaran en la misma casa que el caso índice o que permanecieron en la misma casa por un período mayor a 8 hs diarias durante la semana previa. Como evento de interés primario se consideró la efectividad de la vacuna (protección conferida en un escenario real), determinada mediante la comparación de la tasa de ataque secundaria de varicela en un grupo de niños vacunados vs no-vacunados post exposición. Como evento de interés secundario se consideró la efectividad de la vacuna para protección de enfermedad moderada o intensa, la frecuencia de indicación de la vacuna, el cumplimiento de esta indicación y la tolerancia a la vacuna.

\section{Reclutamiento de los participantes y captura de información}

El estudio contó con la aprobación del Comité de Ética de Investigación de la Facultad de Medicina de la Pontificia Universidad Católica de Chile. Para el reclutamiento de los participantes se contactó telefónicamente a los padres de niños que consultaron por varicela en la Red de Salud UC, y se les preguntó por la existencia de contactos intradomiciliarios que cumplieran con los siguientes criterios de inclusión: edad entre 1 y 12 años, ausencia de historia de varicela y no haber recibido VAV como profilaxis universal. Se excluyeron niños portadores de inmunodeficiencia, usuarios de corticoesteroides sistémicos durante el último mes por más de una semana, receptores de sangre o inmunoglobulinas durante los últimos 3 meses y quienes al momento del contacto telefónico presentaban lesiones compatibles con varicela o que tuvieran una fuente de contagio común. Se invitó a los padres y al niño a participar del estudio, concertándose una reunión con el investigador para la obtención del consentimiento informado. El primer contacto se realizó telefónicamente entre 5 y 7 días después de la consulta del caso índice, de modo de asegurar que el investigador actuara sólo como observador, sin intervenir en la decisión de administración de la vacuna. En los niños reclutados se registraron datos demográficos y clínicos, indicación de la vacuna y el día de su eventual administración. Se realizó un segundo y tercer llamado en los días 14 y 21 desde el inicio de la enfermedad del caso índice, preguntando por la aparición de lesiones compatibles con varicela. Fueron excluidos del estudio los contactos que presentaron varicela dentro de los primeros 10 días desde el inicio de lesiones en el caso índice, puesto que muy probablemente tuvieron una fuente de contagio común que no fue advertida por los padres. Aquellos niños que presentaron lesiones con posterioridad a los 10 días fueron citados a evaluación médica a los 4-5 días de su aparición, momento en que se confirmó la presencia de varicela y se clasificó su intensidad (varicela leve: menos de 50 lesiones, moderada: 51 a 100 lesiones, e intensa: más de 100 lesiones $)^{19}$.

\section{Cálculo de tamaño de muestra y análisis estadístico}

Para el cálculo se utilizó la información aportada por la revisión sistemática realizada por la Colaboración Cochrane (2008), siendo la incidencia de varicela en el grupo que recibió la vacuna hasta cinco días post exposición $18 \%$, y en el grupo que no recibió vacuna post exposición $78 \%{ }^{3}$. Para una confianza de $95 \%$, potencia $80 \%$ y relación expuesto/no-expuesto de $1 / 1$, el tamaño de muestra estimado fue 26 pacientes totales ( 13 por grupo). Como medidas de efecto se calculó el Riesgo Relativo, Reducción del Riesgo Absoluto y Reducción del Riesgo Relativo (este último llamado en otras publicaciones "efectividad de la vacuna"). Como medida de precisión de los resultados se calcularon intervalos de confianza $95 \%$. Para los cálculos se utilizó el programa estadístico OpenEpi 2.3.1.

\section{Resultados}

Se contactó a 120 niños que consultaron por varicela, de los cuales 40 tenían contactos que cumplían criterios de inclusión. Dos contactos rechazaron participar y cuatro fueron excluidos posteriormente por presentar varicela antes del día 10 desde el inicio de lesiones en el caso índice, siendo enrolados 34 niños. En 24 (70,6\%) se indicó la vacuna, administrándose en 15 (62,5\%). Los principales motivos para no administrarla fueron no alcanzar a hacerlo en el plazo recomendado (habitualmente cinco días) y la falta de recursos económicos para comprarla. Trece niños recibieron vacuna Varilrix ${ }^{\circledR}$ (GSK) y dos, Varicela Biken ${ }^{\circledR}$ (SanofiPasteur). Ambas corresponden a vacunas disponibles en los vacunatorios de la red, donde se aplican los procedimientos que aseguran la mantención 
de la cadena de frío, uso de productos vigentes y adecuada administración. Ninguno de los vacunados presentó fiebre; 4/15 (26,7\%) refirieron molestias menores y de corta duración tales como irritabilidad leve y dolor leve en el sitio punción.

La muestra se constituyó de la siguiente manera: 15 niños recibieron VAV como PPE (grupo vacunados) y 19 no la recibieron (grupo no-vacunados). Se excluyó un niño del grupo no-vacunados por pérdida del seguimiento, siendo analizados finalmente 18 no-vacunados. Entre los vacunados, en 12 niños se administró la vacuna dentro de los primeros tres días post exposición y en 3 niños se administró en los días 4 y 5 post exposición (Figura 1). Globalmente, 8/15 (53\%) niños vacunados presentaron varicela, comparado con 16/18 (89\%) no-vacunados. Uno de los niños no-vacunados (y que no desarrolló la enfermedad) fue contacto de un caso índice que desarrolló varicela atenuada por haberse vacunado previamente, situación no contemplada en los criterios de inclusión. En los pacientes que desarrollaron varicela se observó un período de incubación promedio de 14,1 días (rango: 10-21 días). La efectividad de la VAV como PPE para cualquier grado de enfermedad dentro de los cinco días post exposición fue 40\% (IC95\% 1-64\%), aumentando dentro de los tres primeros días post exposición a $44 \%$ (IC95\% -1-69\%). En términos absolutos, la administración de VAV como PPE a 100 contactos susceptibles durante los primeros cinco días post exposición evitaría 36 casos de enfermedad, aumentando a 39 casos si la vacuna se administra durante los primeros tres días post exposición (Tabla 1).

Al comparar la ocurrencia de varicela moderada o intensa vs ausente o leve, la efectividad de la VAV como PPE dentro de los cinco días post exposición fue $63 \%$ (IC95\% 8-85\%), aumentando dentro de los tres primeros días post exposición a 77\% (IC95\% 14-94\%). En términos absolutos, la administración de VAV como PPE a 100 contactos susceptibles durante los primeros cinco días evitaría 45 casos de enfermedad moderada o intensa, aumentando a 55 casos si la vacuna se administra durante los primeros tres días post exposición (Tabla 2).

\section{Discusión}

Los resultados del presente estudio sugieren que la administración de VAV como PPE en contactos domiciliarios es efectiva, reduciendo el riesgo de presentar la enfermedad en $40 \%$, cifra intermedia dentro del rango de $9-100 \%$ reportado en la literatura científica ${ }^{11-21}$. Los estudios que han reportado cifras elevadas de efectividad (80-100\%) corresponden predominantemente a estudios no del todo comparables con el nuestro: tres de ellos se realizaron en contactos hospitalarios ${ }^{12,13,16}$, dos en contactos domiciliarios pero con vacunación en los primeros tres

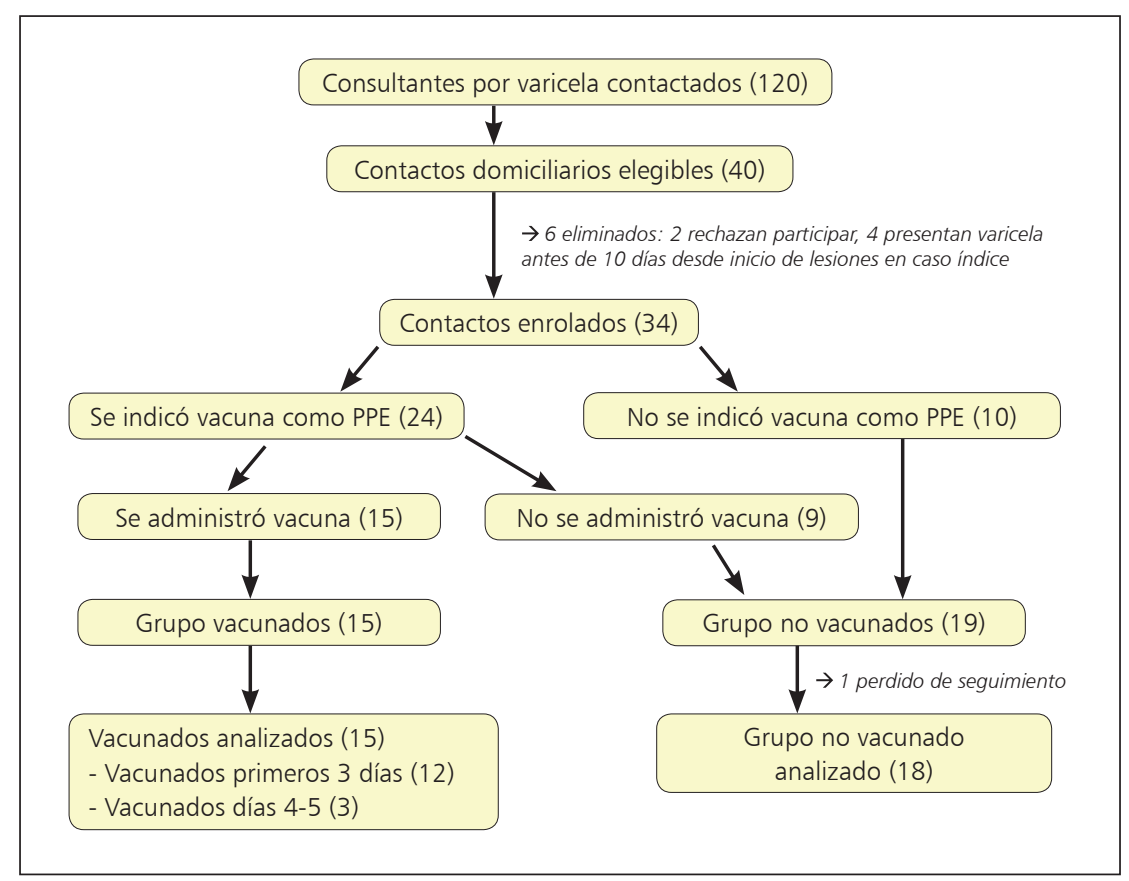

Figura 1. Diagrama de flujo para el reclutamiento de los participantes.

Tabla 1. Tasa de ataque secundaria de varicela y efectividad de la vacuna antivaricela como profilaxis post exposición (varicela presente vs ausente)

\begin{tabular}{|c|c|c|c|c|}
\hline $\begin{array}{l}\text { Profilaxis post } \\
\text { exposición a varicela }\end{array}$ & $\begin{array}{l}\text { Incidencia } \\
\text { varicela }\end{array}$ & $\begin{array}{c}\text { RR } \\
\text { (IC 95\%) }\end{array}$ & $\begin{array}{c}\text { RRR } \\
\text { (Efectividad) } \\
\text { (IC 95\%) }\end{array}$ & $\begin{array}{c}\text { RRA } \\
\text { (IC 95\%) }\end{array}$ \\
\hline $\begin{array}{l}\text { Primeros } 5 \text { días } \\
\text { Vacunados } \\
\text { No-vacunados }\end{array}$ & $\begin{array}{r}8 / 15(53 \%) \\
16 / 18(89 \%)\end{array}$ & $\begin{array}{c}0,60 \\
(0,36-0,99)\end{array}$ & $\begin{array}{c}40 \% \\
(1 \%-64 \%)\end{array}$ & $\begin{array}{c}36 \% \\
(6 \%-65 \%)\end{array}$ \\
\hline $\begin{array}{l}\text { Primeros } 3 \text { días } \\
\text { Vacunados } \\
\text { No-vacunados }\end{array}$ & $\begin{array}{r}6 / 12(50 \%) \\
16 / 18(89 \%)\end{array}$ & $\begin{array}{c}0,56 \\
(0,31-1,01)\end{array}$ & $\begin{array}{c}44 \% \\
(-1 \%-69 \%)\end{array}$ & $\begin{array}{c}39 \% \\
(7 \%-71 \%)\end{array}$ \\
\hline
\end{tabular}

RR: riesgo relativo, RRR: reducción el riesgo relativo, RRA: reducción del riesgo absoluto. IC: Intervalo de confianza.

Tabla 2. Tasa de ataque secundaria de varicela y efectividad de la vacuna antivaricela como profilaxis post exposición (varicela moderada o intensa vs ausente o leve)

\begin{tabular}{|c|c|c|c|c|}
\hline $\begin{array}{l}\text { Profilaxis post } \\
\text { exposición a varicela }\end{array}$ & $\begin{array}{l}\text { Incidencia } \\
\text { varicela } \\
\text { (moderada o } \\
\text { intensa) }\end{array}$ & $\begin{array}{c}\text { RR } \\
\text { (IC 95\%) }\end{array}$ & $\begin{array}{c}\text { RRR } \\
\text { (Efectividad) } \\
\text { (IC 95\%) }\end{array}$ & $\begin{array}{c}\text { RRA } \\
\text { (IC 95\%) }\end{array}$ \\
\hline $\begin{array}{l}\text { Primeros } 5 \text { días } \\
\text { Vacunados } \\
\text { No-vacunados* }\end{array}$ & $\begin{array}{l}4 / 15(27 \%) \\
10 / 14(71 \%)\end{array}$ & $\begin{array}{c}0,37 \\
(0,15-0,92)\end{array}$ & $\begin{array}{c}63 \% \\
(8 \%-85 \%)\end{array}$ & $\begin{array}{c}45 \% \\
(12 \%-77 \%)\end{array}$ \\
\hline $\begin{array}{l}\text { Primeros } 3 \text { días } \\
\text { Vacunados } \\
\text { No-vacunados* }\end{array}$ & $\begin{array}{l}2 / 12(17 \%) \\
10 / 14(71 \%)\end{array}$ & $\begin{array}{c}0,23 \\
(0,06-0,86)\end{array}$ & $\begin{array}{c}77 \% \\
(14 \%-94 \%)\end{array}$ & $\begin{array}{c}55 \% \\
(23 \%-86 \%)\end{array}$ \\
\hline
\end{tabular}

RR: riesgo relativo, RRR: reducción el riesgo relativo, RRA: reducción del riesgo absoluto, IC: Intervalo de confianza. *Se analizó severidad en 14/18 niños. No se analizaron 4 niños: 1 no estuvo disponible para evaluar severidad y en 3 se utilizó aciclovir (altera evolución natural de varicela y, por lo tanto, su severidad). 
días $^{14,18}$ y uno en un refugio de niños sin hogar ${ }^{20}$. Un estudio reciente realizado en Argentina, de cohorte comparado con grupo histórico, mostró una efectividad de 87,5\% a los 2 días y $83,7 \%$ a los cinco días ${ }^{21}$. Dos ensayos clínicos aleatorios incluidos en la revisión Cochrane reportaron una efectividad de 90 y $100 \%$, respectivamente, a los tres días ${ }^{17,18}$ y de $67 \%$ a los cinco días ${ }^{17}$. Una efectividad similar a la de nuestro estudio fue reportada por Salzman et al., en 10 contactos intradomiciliarios entre 14 meses y 12 años de edad, en un diseño sin grupo control ${ }^{15}$. Los resultados menos alentadores fueron obtenidos en otro ensayo clínico aleatorio en que la eficacia reportada fue de 9,1\% para toda enfermedad, y de $87,5 \%$ para enfermedad moderada a intensa ${ }^{19}$. Esta baja eficacia global obedece en parte a la baja tasa de varicela observada en los niños no-vacunados (45\%), cifra que difiere en forma importante de la conocida alta transmisibilidad de la varicela en contactos cercanos; además, como comentan los autores de la revisión Cochrane, se debe a que un gran porcentaje (59\%) de los vacunados recibió la vacuna en los días 4 y 5 post exposición ${ }^{19}$; en cambio, en los otros dos estudios incluidos en esta revisión, el porcentaje de los vacunados después de las $72 \mathrm{~h}$ fue muy bajo ${ }^{17,18}$.

Se ha planteado la hipótesis que diferencias en las vacunas utilizadas podrían explicar los distintos valores de eficacia y efectividad observados. El ensayo clínico aleatorio que mostró una eficacia de $90 \%$ utilizó una vacuna con más de 4.000 unidades formadoras de placas (ufp) ${ }^{17}$, mientras que los otros en general han usado vacunas de alrededor de 1.000 a 3.000 ufp $^{3,12-16,18,19}$. En la misma línea, un estudio alemán que analizó la protección de distintas marcas de vacunas usadas pre exposición en brotes de varicela en jardines infantiles mostró cifras variables según la vacuna utilizada ${ }^{22}$; sin embargo, la metodología de este estudio fue criticada ${ }^{23}$. La vacuna utilizada en un estudio argentino fue la misma que recibió la mayoría de los niños de nuestro estudio, y el diseño fue similar ${ }^{21}$, por lo que este factor no explicaría la menor eficacia observada por nosotros.

Con relación al momento de la vacunación, se observó un discreto aumento de la efectividad en los primeros tres días (44\%) comparado a los primeros cinco días (40\%), hecho que puede explicarse por el reducido número de niños vacunados en los días 4 y 5 en nuestra serie (sólo tres niños). Como fuera mencionado, numerosos estudios han mostrado una mayor eficacia en el uso precoz de la vacuna $^{12,13,17,18,21}$. A pesar de la moderada efectividad global de la VAV como PPE observada en nuestro estudio, la protección contra formas más intensas fue mayor: $63 \%$ de efectividad para prevenir enfermedad moderada o intensa para el grupo completo y $77 \%$ para los vacunados en los primeros tres días. Debe hacerse notar que este resultado se obtuvo aplicando la definición de enfermedad intensa usada por Mor ${ }^{19}$, quien la define como aquella con más de 100 lesiones. Por desgracia no existe consenso en la calificación de la intensidad de la varicela; textos importantes de pediatría describen que una varicela típica presenta entre 250 y 500 lesiones $^{24}$, otros definen varicela intensa como aquella con fiebre alta y prolongada y/o con presencia de complicaciones ${ }^{11,25}$ y otros como aquella con más de 500 lesiones $^{20}$. Usando esta última definición, la cifra de protección contra enfermedad intensa sería aún mayor.

La vacuna fue bien tolerada, registrándose un escaso número de efectos adversos, los que fueron en su mayoría leves (dolor en el sitio de inyección e irritabilidad), coincidiendo con lo reportado en estudios de vigilancia post vacuna $^{26,27}$. Otras reacciones adversas que se han descrito con menor frecuencia en relación al uso de esta vacuna tales como exantema, herpes zoster o eventos neurológicos no fueron registradas en esta serie. Destaca el alto porcentaje de niños que no se vacunaron a pesar de haber recibido la indicación $(37,5 \%)$, ya fuese por no alcanzar a hacerlo en el plazo indicado o por motivos económicos; al respecto, la gratuidad en la vacuna o su cobertura por los sistemas previsionales o seguros de salud podrían ayudar a mejorar estas cifras. En situaciones en que no se pueda usar la vacuna o la consulta ocurra más allá del período en que ella está indicada, puede considerarse el uso de aciclovir profiláctico ${ }^{4,7-9}$.

Nuestro estudio presenta algunas limitaciones inherentes a su diseño. Si bien su tamaño de muestra fue calculado en base a la mejor evidencia disponible ${ }^{3}$, el grado de precisión de nuestras estimaciones fue bajo, hecho que se explica por el gran tamaño de efecto reportado por la revisión sistemática utilizada para el cálculo del tamaño de muestra. Ello tampoco permitió establecer comparaciones de subgrupos (vacunación en primeros tres días versus 4-5 días, formulaciones de vacuna diferentes, etc.). Asimismo, el antecedente de haber desarrollado varicela previa se obtuvo por anamnesis, sin confirmación serológica. Por el contrario, entre sus ventajas se encuentran el contar con un grupo control (contactos domiciliarios no-vacunados), el haber evaluado los casos con lesiones por un observador entrenado, y el haber sido realizado en condiciones reales (a diferencia de los estudios de eficacia) en un país donde esta vacuna no está incluida en el esquema de vacunación obligatorio, de modo que la mayoría de los casos índices corresponden a varicela "habitual" (no atenuada por vacuna).

La inclusión en el análisis del niño cuyo caso índice presentó varicela atenuada por haber sido vacunado previamente se basa en que esta situación no se consideró desde un principio como un criterio de exclusión, por lo tanto, dicho antecedente no fue evaluado sistemáticamente. En vista que este niño no desarrolló varicela, su inclusión en el análisis va en contra de la hipótesis planteada para el estudio, hecho que otorga mayor validez a los resultados. Por 
otro lado, si se asume que el niño perdido del seguimiento (excluido del análisis) no hubiera presentado varicela (peor escenario para la hipótesis planteada para el estudio), la efectividad de la VAV como PPE para cualquier grado de enfermedad se reduciría a 37\% (IC95\%-6-62\%) para los vacunados en los primeros cinco días y $41 \%$ (-8-67\%) para los vacunados en los primeros tres días.

En conclusión, el presente estudio sugiere que el uso de VAV como PPE en contactos domiciliarios confiere una protección moderada para evitar la enfermedad, y una buena protección para evitar varicela moderada o intensa. Esto es concordante con la información publicada a la fecha, la que si bien muestra resultados variables en la reducción del riesgo de enfermar, ha sido consistente en la efectividad de esta medida en atenuar la enfermedad. No obstante ello, aún es necesario contar con nuevos estudios, de mayor tamaño que el presente, y que puedan enfocarse en aspectos no aclarados tales como el rol de las distintas formulaciones, así como el uso de una mejor definición de intensidad. En estos estudios debe establecerse a priori las características del caso índice, asegurándose que ellos constituyan casos de varicela natural y no atenuada por vacuna, de modo de poder atribuir el resultado a la PPE y no a otras posibles causas.

\section{Resumen}

Introducción: Los estudios de eficacia y efectividad de la vacuna antivaricela (VAV) como profilaxis post exposición (PPE) han mostrado resultados disímiles en cuanto a la reducción del riesgo de enfermar, y algo más consistentes en su capacidad de atenuar la enfermedad. Objetivo: Evaluar la efectividad de la VAV como PPE utilizada en contactos domiciliarios. Material y Métodos: Se realizó el seguimiento post exposición de 33 contactos domiciliarios de casos de varicela, de los cuales 15 recibieron VAV como PPE y 18 no la recibieron. Se determinó clínicamente el desarrollo de enfermedad y la intensidad de la misma. Resultados: La tasa de ataque secundario fue 53\% en vacunados y $89 \%$ en no-vacunados, siendo la efectividad global de la VAV como PEP 40\% (IC95\% 1\%-64\%). La efectividad para prevenir enfermedad moderada o intensa fue $63 \%$ (IC95\% 8-85\%) en el grupo completo y $77 \%$ (IC95\% 14-94\%) en los niños vacunados durante los primeros tres días post exposición. La tolerancia a la vacuna fue aceptable, con un bajo número de reacciones adversas, todas leves. Conclusión: Los resultados sugieren que la VAV es efectiva como PPE en contactos domiciliarios, especialmente para reducir la intensidad de la enfermedad.

\section{Referencias bibliográficas}

1.- English R. Varicella. Pediatr Rev 2003; 24: 372-9.

2.- Abarca K, Hirsch T, Potin M, Perret C, Zamorano J, González C, et al. Complicaciones en niños con varicela en cuatro hospitales de Santiago- Chile: Espectro clínico y estimación de costos directos. Rev Med Chile 2001; 129 : 397-404.

3.- Macartney K, McIntyre P. Vaccines for post-exposure prophylaxis against varicella (chickenpox) in children and adults. Cochrane Database of Systematic Reviews 2008, Issue 3.Art.No.: CD001833. DOI:10.1002/14651858. CD001833.pub2.

4.- Abarca K. Varicela: Indicaciones actuales de tratamiento y prevención. Rev Chilena Infectol 2004; 21 (Supl1): S20-3.

5.- Abarca K. Vacuna anti-varicela. Rev Chilena Infectol 2006; 23: 56-9.

6.- Committee on Infectious Diseases. Prevention of varicella: Recommendations for use of varicella vaccines in children, including a recommendation for a routine 2-dose varicella immunization schedule. Pediatrics 2007; 120: 221-31.

7.- Asano Y, Yoshikawa T, Suga S, Kobayashi I, Nakashima T, Yazaki T, et al. Postexposure prophylaxis of varicella in family contact by oral acyclovir. Pediatrics. 1993; 92 : 219-22.
8.- Lin T, Hauang Y, Ning H, Hsueh C. Oral acyclovir prophylaxis of varicella after intimate contact. Pediatr Infect Dis J 1997; 16: $1162-5$.

9.- Goldstein G, García A, Aguirre A, Dieser P, Gaivironsky R, Polack N. Aciclovir oral para profilaxis posexposición familiar a varicela. Arch Argent Pediatr 2004; 102 (1): 13-7.

10.- Centers for Disease Control and Prevention. Prevention of varicella: recommendations of the Advisory Committee on Immunization Practices (ACIP), 2007. MMWR Recomm Rep 2007; 22 (RR-4): 1-40.

11.- Brotons M, Campins M, Méndez L, Juste C, Rodrigo J, Martínez X, et al. Effectiveness of varicella vaccines as postexposure prophylaxis. Pediatr Infect Dis J 2010; 29: 10-3.

12.- Takahashi M, Otsuka T, Okuno Y, Asano Y, Yasaki T. Live vaccine used to prevent the spread of varicella in children in hospital. Lancet 1974; 30; 2 (7892): 1288-90.

13.- Asano Y, Hirose S, Iwayama S, Miyata T, Yasaki T, Takahashi M. Protective effect of immediate inoculation of a live varicella vaccine in household contacts in relation to the viral dose and interval between exposure and vaccination. Biken J 1982; 25: 43-5.

14.- Katsushima N, Yasaki N, Sakamoto M. Effect and follow-up on varicella vaccine. Biken J 1984; 27: 51-8.

15.- Salzman M, García C. Postexposure varicella vaccination in siblings of children with active varicella. Pediatr Infect Dis J 1998; 17: 256-7.

16.- Nagamuna Y, Osawa S, Takahashi R. Clinical application of a live varicella vaccine (Oka strain) in a hospital. Biken J 1984; 27: 59-61.

17.- Arbeter A, Starr S, Plotkin S. Varicella vaccine studies in healthy children and adults. Pediatrics 1986; 78 (Suppl): 748-56.

18.- Asano Y, Nakayama H, Yazaki T, Kato R, Hirose S. Protection against varicella in family contacts by immediate inoculation with live varicella vaccine. Pediatrics 1977; 59: 3-7.

19.- Mor M, Harel L, Kahan E, Amir J. Efficacy of postexposure immunization with live attenuated varicella vaccine in the household setting- a pilot study. Vaccine 2004; 23: 325-8.

20.- Watson B, Seward J, Yang A, Witte P, Lutz J, Chan C. Postexposure effectiveness of varicella vaccine. Pediatrics 2000; 105: 84-8.

21.- Gentile A, Marco Del Pont J, Martínez E, Pueta G, Walter J, Catrillón S. Efectividad de la vacuna antivaricela zoster como profilaxis post exposición. Arch Argent Pediatr 2002; 100: 25-30.

22.- Spackova M, Wiese- Posselt, Denhert M, Matysiak-Klose D, Heiniger U, Siedler A. Comparative varicella vaccine effectiveness during outbreaks in day-care centers. Vaccine 2010; 28: 686-91.

23.- Marchetti F. Letter to the editor. Vaccine 2010; 31: 4859-60.

24.- American Academy of Pediatrics. Varicela- 
Zoster Infections. In: Pickering LK, Baker CJ, Kimberlin DW; Long SS, eds. Redbook: 2012 Report of the Committee on Infectious Diseases. $29^{\text {th }}$ ed. ELK Grove Village, IL: American Academy of Pediatrics; 2012: 714-27.

25.- Miron D, Lavi I, Kitov R, Hendelr A. Vaccine effectiveness and severity of varicella among previously vaccinated children during outbreaks in day-care centers with low vaccination coverage. Pediatr Infect Dis J 2005; 24: 233-6.

26.- Goulleret N, Mauvisseau E, Essevaz-Roulet M, Quinlivan M, Breuer J. Safety profile of varicela virus vaccine (Oka/Merck): Five-year results of the European Varicella Zoster Virus Identification Program (EU VZVIP). Vaccine 2010; 28: 5878-82.

27.- Galea S, Sweet A, Beninger P, Steinberg S, LaRussa P, Gershon A. The safety profile of varicella vaccine: a 10 -year review. J Infect Dis 2008; 197 (Suppl 2): S165-9. 